OPEN ACCESS

Edited by:

Saad J. Taj-Aldeen,

Hamad Medical Corporation, Qatar

Reviewed by:

Maurizio Sanguinetti,

Catholic University of the Sacred

Heart, Italy

Guillaume Desoubeaux,

Université de Tours, France

*Correspondence:

Anna F. Lau

Anna.Lau@nih.gov

${ }^{+}$Present address:

Jeremy J. Gillbreath,

ICON plc, North Wales, PA,

United States

Susan Butler-Wu,

Department of Clinical Pathology,

University of Southern California,

Los Angeles, CA, United States

Audrey N. Schuetz,

Division of Clinical Microbiology, Mayo

Clinic, Rochester, MN, United States

Specialty section:

This article was submitted to Fungi and Their Interactions,

a section of the journal

Frontiers in Microbiology

Received: 17 July 2019

Accepted: 26 August 2019

Published: 20 September 2019

Citation:

Lau AF, Walchak RC, Miller HB,

Slechta ES, Kamboj K, Riebe K,

Robertson $A E$, Gillbreath $\mathrm{JJ}$,

Mitchell KF, Wallace MA, Bryson AL,

Balada-Llasat J-M, Bulman A,

Buchan BW, Burnham C-AD,

Butler-Wu S, Desai U, Doern CD,

Hanson KE, Henderson CM,

Kostrzewa M, Ledeboer NA, Maier T,

Pancholi P, Schuetz AN, Shi G,

Wengenack NL, Zhang SX,

Zelazny AM and Frank KM (2019)

Multicenter Study Demonstrates

Standardization Requirements

for Mold Identification by MALDI-TOF

MS. Front. Microbiol. 10:2098.

doi: 10.3389/fmicb.2019.02098

\section{Multicenter Study Demonstrates Standardization Requirements for Mold Identification by MALDI-TOF MS}

Anna F. Lau' ${ }^{*}$, Robert C. Walchak ${ }^{2}$, Heather B. Miller ${ }^{3}$, E. Susan Slechta', Kamal Kamboj ${ }^{5}$, Katherine Riebe ${ }^{6}$, Amy E. Robertson ${ }^{7}$, Jeremy J. Gilbreath ${ }^{8 \dagger}$, Kaitlin F. Mitchell ${ }^{9}$, Meghan A. Wallace ${ }^{9}$, Alexandra L. Bryson ${ }^{10}$, Joan-Miquel Balada-Llasat ${ }^{5}$, Amanda Bulman ${ }^{11}$, Blake W. Buchan ${ }^{6}$, Carey-Ann D. Burnham ${ }^{9}$, Susan Butler-Wu ${ }^{81}$, Uma Desai ${ }^{1}$, Christopher D. Doern ${ }^{10}$, Kimberly E. Hanson ${ }^{4,12}$, Christina M. Henderson ${ }^{1}$, Markus Kostrzewa ${ }^{11}$, Nathan A. Ledeboer ${ }^{6}$, Thomas Maier ${ }^{11}$, Preeti Pancholi ${ }^{5}$, Audrey N. Schuetz ${ }^{7 \dagger}$, Gongyi Shi ${ }^{11}$, Nancy L. Wengenack ${ }^{2}$, Sean X. Zhang ${ }^{3}$, Adrian M. Zelazny ${ }^{1}$ and Karen M. Frank ${ }^{1}$

\begin{abstract}
' Department of Laboratory Medicine, Clinical Center, National Institutes of Health, Bethesda, MD, United States, ${ }^{2}$ Division of Clinical Microbiology, Mayo Clinic, Rochester, MN, United States, ${ }^{3}$ Department of Pathology, Johns Hopkins University School of Medicine, Baltimore, MD, United States, ${ }^{4}$ ARUP Institute for Clinical and Experimental Pathology, Salt Lake City, UT, United States, ${ }^{5}$ Department of Pathology, The Ohio State University Wexner Medical Center, Columbus, OH, United States, ${ }^{6}$ Department of Pathology, Medical College of Wisconsin, Milwaukee, WI, United States, ${ }^{7}$ Clinical Microbiology Laboratory, Weill Cornell Medical Center/New York Presbyterian Hospital, New York, NY, United States, ${ }^{8}$ Department of Laboratory Medicine, University of Washington, Seattle, WA, United States, ${ }^{9}$ Department of Pathology and Immunology, Washington University School of Medicine, St. Louis, MO, United States, ${ }^{10}$ Department of Pathology, Virginia Commonwealth University School of Medicine, Richmond, VA, United States, " ${ }^{11}$ Bruker Daltonics, Inc., Billerica, MA, United States, ${ }^{12}$ Department of Pathology, Division of Clinical Microbiology, The University of Utah, Salt Lake City, UT, United States
\end{abstract}

Objectives: Rapid and accurate mold identification is critical for guiding therapy for mold infections. MALDI-TOF MS has been widely adopted for bacterial and yeast identification; however, few clinical laboratories have applied this technology for routine mold identification due to limited database availability and lack of standardized processes. Here, we evaluated the versatility of the $\mathrm{NIH}$ Mold Database in a multicenter evaluation.

Methods: The NIH Mold Database was evaluated by eight US academic centers using a solid media extraction method and a challenge set of 80 clinical mold isolates. Multiple instrument parameters important for spectra optimization were evaluated, leading to the development of two specialized acquisition programs $(\mathrm{NIH}$ method and the Alternate-B method).

Results: A wide range in performance (33-77\%) was initially observed across the eight centers when routine spectral acquisition parameters were applied. Use of the $\mathrm{NIH}$ or the Alternate-B specialized acquisition programs, which are different than those used routinely for bacterial and yeast spectral acquisition (MBT_AutoX), in combination with optimized instrument maintenance, improved performance, illustrating that acquisition parameters may be one of the key limiting variable in achieving successful performance.

Conclusion: Successful mold identification using the NIH Database for MALDI-TOF MS on Biotyper systems was demonstrated across multiple institutions for the first 
time following identification of critical program parameters combined with instrument optimization. This significantly advances our potential to implement MALDI-TOF MS for mold identification across many institutions. Because instrument variability is inevitable, development of an instrument performance standard specific for mold spectral acquisition is suggested to improve reproducibility across instruments.

Keywords: mold, filamentous fungi, MALDI-TOF MS, rapid, identification

\section{INTRODUCTION}

Rapid bacterial and yeast identification by matrix-assisted laser desorption ionization-time of flight mass spectrometry (MALDITOF MS) has revolutionized microbiology laboratory practices (Tan et al., 2012; Huang et al., 2013; Lacroix et al., 2014). Studies evaluating MALDI-TOF MS for mold identification have been published, but only a few clinical laboratories have applied this technology in routine practice. Limiting factors have included (1) poor performance of manufacturer's databases leading to a heavy dependency on laboratory-developed databases (including online databases such as the Mass Spectrometry Identification Platform (MSI) (Normand et al., 2017) and MicrobeNet from the Centers for Disease Control and Prevention (CDC) (Center for Disease Control and Prevention [CDCP], 2019); (2) lack of standardized processing methods (Alanio et al., 2011; Cassagne et al., 2011; Theel et al., 2011; Alshawa et al., 2012; De Carolis et al., 2012; Del Chierico et al., 2012; Schrodl et al., 2012; de Respinis et al., 2013; Lau et al., 2013; L'Ollivier et al., 2013; Nenoff et al., 2013; Normand et al., 2013; Barker et al., 2014; Becker et al., 2014; Gautier et al., 2014; Ranque et al., 2014; Sitterle et al., 2014; Dolatabadi et al., 2015; Triest et al., 2015; Sleiman et al., 2016); and (3) the absence of an FDA-cleared mold database until recent release of the VITEK MS 3.0 (bioMerieux, Inc., Durham, NC, United States) which includes representation of 47 filamentous fungi (United States Food and Drug Administration, 2017). Many clinical laboratories worldwide, however, utilize either the Bruker MALDI Biotyper (MBT) system for which the Filamentous Fungal Database has not been cleared by the FDA, or the older VITEK MS 2.0 which lacks the mold database due to the time needed for test system reverification and/or software compatibility restraints.

Since 2012, the National Institutes of Health (NIH) Clinical Center has applied MALDI-TOF MS for the routine clinical identification of molds for patient care directly from solid media using the NIH Mold Database (available publicly since 2013) to supplement that of the Bruker MBT system (Lau et al., 2013). To investigate the broader utility of the NIH Mold Database beyond our institution, a multicenter study including eight US academic institutions was conducted. Unlike multicenter bacterial and yeast studies for which excellent inter- and intra-laboratory reproducibility has been demonstrated (Westblade et al., 2015; Wilson et al., 2017), this study has highlighted significant performance variability between instruments regardless of acquisition parameters and demonstrated that development of a specific mold standard is required for successful use of MALDITOF MS for mold identification.

\section{MATERIALS AND METHODS}

\section{Isolates}

Eighty clinical isolates (10 from each of the eight original participating centers - National Institutes of Health, Bethesda, MD; Mayo Clinic, Rochester, MN; The Ohio State University Wexner Medical Center, Columbus, OH; ARUP Institute for Clinical and Experimental Pathology, Salt Lake City, UT; Medical College of Wisconsin, Milwaukee, WI; University of Washington, Seattle, WA; Johns Hopkins University School of Medicine, Baltimore, MD; and Weill Cornell Medical Center/New York Presbyterian Hospital, New York, NY, United States) representing a diverse group of common clinical molds and less common organisms were studied (Table 1). An additional two centers (Washington University and Virginia Commonwealth University) assisted with testing at the final stages of the study. All 80 isolates were shipped to the NIH, blinded, subcultured onto Sabouraud Dextrose Agar (SDA) slants, and then redistributed to each of the centers. Upon receipt, isolates were subcultured onto SDA prior to protein extraction performed at each of the individual laboratories. To effectively challenge the specificity of the NIH Mold Database, some isolates that were not well-represented in the database were purposefully selected. Gold standard identification for each isolate included macroscopic and microscopic morphologies with confirmation by Sanger sequencing of the ITS1-2 (White et al., 1990), when necessary. Species-level identification was not possible for all isolates as the ITS region was unable to provide sufficient discriminatory power following MM18 guidelines provided by the Clinical Laboratory Standards Institute (Clinical and Laboratory Standards Institute [CLSI], 2008).

\section{Protein Extraction and MALDI-TOF MS}

Extraction was performed using the NIH solid media extraction method as described previously (Lau et al., 2013) and in this video $^{1}$. Spectra were collected on MicroFlex LT instruments (Bruker Daltonics, Inc.). The same versions of the NIH database and Biotyper MBT (including the Filamentous Fungal Database) were applied for spectral analysis in all centers. Programing instructions for the $\mathrm{NIH}$ and Alternate-B spectral acquisition methods are provided in Supplementary Figures 1, 2.

\footnotetext{
${ }^{1}$ https://www.dropbox.com/s/gt4mfk5nj5y9qif/ NIHMoldMALDIExtractionVideo.mov
} 
TABLE 1 | List of mold isolates used in study $(n=80)$.

\begin{tabular}{|c|c|c|c|c|}
\hline Hyaline $(n=36)$ & Dematiaceous ( $n=22$ ) & Mucorales $(n=9)$ & Dermatophytes $(n=7)$ & Dimorphic fungi $(n=6)$ \\
\hline Aspergillus fumigatus (3) & Aureobasidium pullulans (1) & Lichtheimia corymbifera (2) & Trichophyton rubrum (2) & Histoplasma capsulatum (2) \\
\hline Aspergillus glaucus group (1) & Chaetomium spp. (2) & Mucor spp. (1)* & Trichophyton spp. (2)* & Sporothrix schenckii (2) \\
\hline Aspergillus sydowii (1)* & Cladosporium spp. (1) & Syncephalastrum spp. (2)* & & \\
\hline Aspergillus terreus (2)* & Epicoccum spp. (1) & & & \\
\hline Aspergillus ustus (1)* & Exophiala dermatitidis (2)* & & & \\
\hline Eutypella spp. (1) & Ochroconis spp. (2)* & & & \\
\hline Fusarium oxysporum (1) & Phaeoacremonium spp. (1) & & & \\
\hline Fusarium solani (1)* & Phialophora spp. (1) & & & \\
\hline Fusarium spp. (2) & Phoma spp. (2) & & & \\
\hline Fusarium verticillioides (1) & Scedosporium apiospermum (1)* & & & \\
\hline Hormographiella spp. (1) & Scedosporium dehoogii (1) & & & \\
\hline \multicolumn{5}{|l|}{ Myrmecridium spp. (1) } \\
\hline \multicolumn{5}{|l|}{ Paecilomyces saturatum (1) } \\
\hline \multicolumn{5}{|l|}{ Paecilomyces variotii (1) } \\
\hline \multicolumn{5}{|l|}{ Penicillium spp. (2) } \\
\hline \multicolumn{5}{|l|}{ Purpureocillium lilacinum (2)* } \\
\hline \multicolumn{5}{|l|}{ Rasamsonia argillacea (3)* } \\
\hline \multicolumn{5}{|l|}{ Scopulariopsis brevicaulis (1) } \\
\hline Trichoderma spp. (1) & & & & \\
\hline
\end{tabular}

*Subset of representative strains used in later analyses for comparison of spectral acquisition methods ( $n=17 ; \mathrm{NIH}$ mold study strains $7,11,18,20,22-24,26,28-29$, $45,64,66,67,71,76$, and A. ustus CBS $261.67 T$ control strain).

\section{Study Design}

Instrument model, software, database types and versions, isolates, and identification criteria were identical across the testing centers. Operators at each center varied in mass spectrometry skill level and experience, reflecting the diversity observed in clinical laboratories. Three major investigative arms were conducted sequentially - (1) evaluation of database performance using routine spectral acquisition programs set at each institution; (2) a pilot evaluation of database performance following optimization of instrument settings; and (3) evaluation of database performance using different spectral acquisition methods (NIH and Alternate-B). After analysis at each institution, extracts were frozen and transferred to the NIH for further testing. Statistical analyses were performed using Fisher's exact test and two-tailed $p$-value ${ }^{2}$.

\section{RESULTS}

\section{Wide Variation in Performance Between Centers Using Routine Spectral Acquisition Programs}

Following organism distribution with a set of written extraction procedural instructions to each center, species-level identification (score $\geq 2.0$ ) ranged from 32 to $77 \%$ across the eight original centers; Center 1 (NIH instrument) performed significantly better than others (Table 2). Without compromising accuracy,

\footnotetext{
${ }^{2}$ www.graphpad.com
}

identification improved to $53-85 \%$ if the score acceptability threshold was lowered to $\geq 1.7$. Nine (11\%) isolates (Acremonium spp., Aureobasidium pullulans, Chaetomium spp., Metarhizium spp., Penicillium spp., Phialophora spp., Phoma spp., Sporothrix schenckii, and Syncephalastrum spp.) failed to identify by the $\mathrm{NIH}$ database across all eight centers consistent with the database containing limited strain representation of some molds (maximum performance capacity of $89 \%$ from this set of 80 isolates). We purposefully created a challenge set beyond common and highly represented molds. No misidentifications were observed with the NIH Mold Database; a few misidentifications were noted with the Bruker MBT Database (Table 2).

To determine if performance variation may be due to misinterpretation of initial instructions, study participants were asked to review a video of the protein extraction procedure after which fresh subcultures of the 80 isolates were reextracted at each site. Overall performance, however, did not improve with Center 1 continuing to perform significantly better than the other nine instruments at Centers 2-8 (Table 3). Frozen protein extracts from each site were sent to the NIH to assess the quality of the protein extraction technique. Despite frozen storage of extracts for up to 9 months, spectra acquired on the NIH instrument (Center 1) were equivalent to or better than results obtained originally using fresh extracts on seven of nine non-NIH instruments (Table 3). This indicated that protein extraction quality was not the limiting factor, suggesting that an important parameter contributing to result variability was differences between instruments. 
TABLE 2 | Initial Comparison of Mold Identification Performance at Eight Different Institutions ( $n=80$ isolates).

\begin{tabular}{|c|c|c|c|c|c|c|c|c|c|c|}
\hline \multirow[t]{2}{*}{ Center } & \multicolumn{3}{|c|}{ NIH Mold Database (\% accuracy) } & \multicolumn{3}{|c|}{$\begin{array}{l}\text { Bruker MBT Database }{ }^{\#} \text { (including } \\
\text { Filamentous Fungal Database; \% } \\
\text { accuracy) }\end{array}$} & \multicolumn{2}{|c|}{$\begin{array}{c}p \text {-Value (NIH vs. Bruker } \\
\text { MBT database) }\end{array}$} & \multicolumn{2}{|c|}{$\begin{array}{c}\text { p-Value (Center } 1 \text { vs. } \\
\text { Centers 2-8; NIH } \\
\text { database) }\end{array}$} \\
\hline & $\geq 2.0$ & $\geq 1.7$ & $\begin{array}{l}\text { Mis } \\
\text { identified }\end{array}$ & $\geq 2.0$ & $\geq 1.7$ & Misidentified & $\geq 2.0$ & $\geq 1.7$ & $\geq 2.0$ & $\geq 1.7$ \\
\hline 1 & 77 & 85 & 0 & 41 & 60 & 4 & $<0.0001$ & 0.0006 & NA & NA \\
\hline $2 A^{*}$ & 33 & 53 & 0 & 3 & 19 & 1 & $<0.0001$ & $<0.0001$ & $<0.0001$ & $<0.0001$ \\
\hline 3 & 46 & 68 & 0 & 40 & 50 & 4 & 0.5 & 0.04 & $<0.0001$ & 0.02 \\
\hline 4 & 51 & 71 & 0 & 6 & 19 & 1 & $<0.0001$ & $<0.0001$ & 0.0008 & 0.05 \\
\hline 5 & 53 & 65 & 0 & 33 & 49 & 0 & 0.02 & 0.05 & 0.002 & 0.006 \\
\hline 6 & 54 & 74 & 0 & 5 & 18 & 1 & $<0.0001$ & $<0.0001$ & 0.003 & 0.1 \\
\hline 7 & 32 & 56 & 0 & 32 & 54 & 5 & 1.000 & 0.7 & $<0.0001$ & 0.0006 \\
\hline 8 & 33 & 61 & 0 & 11 & 29 & 3 & 0.001 & 0.0002 & $<0.0001$ & 0.001 \\
\hline
\end{tabular}

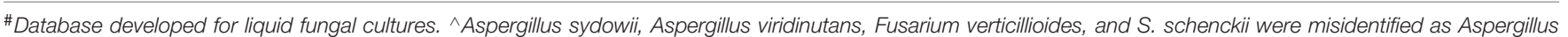

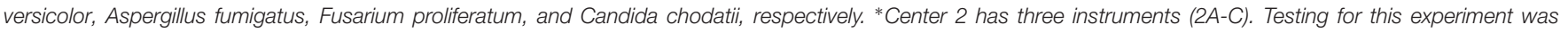
performed on instrument 2A only. Instruments 2B and 2C were tested in later phases of the study and are discussed in other Figures.

TABLE 3 | Evaluation of instructional video and extract quality ( $n=80$ isolates).

\begin{tabular}{|c|c|c|c|c|}
\hline \multirow[t]{2}{*}{ Center } & \multicolumn{2}{|c|}{ NIH Mold Database at Score $\geq 2.0$ ( $\%$ accuracy $)$} & \multirow[b]{2}{*}{$\begin{array}{c}p \text {-Value Fresh extraction } \\
\text { following instructional video } \\
\text { Original center (Center } 1 \text { vs. } \\
\text { Centers 2-8) }\end{array}$} & \multirow[b]{2}{*}{$\begin{array}{l}p \text {-Value Frozen extracts run on } \\
\text { original center instrument vs. } \\
\text { NIH instrument (after } \\
1-9 \text { months frozen) }\end{array}$} \\
\hline & $\begin{array}{l}\text { Original } \\
\text { center (video } \\
\text { procedure) }\end{array}$ & $\begin{array}{l}\text { Frozen extracts analyzed on } \\
\mathrm{NIH} \text { instrument (time stored } \\
\text { at }-20^{\circ} \mathrm{C} \text { prior to analysis) }\end{array}$ & & \\
\hline 1 & 71 & 61 (9 months) & NA & 0.4 \\
\hline $2 A^{*}$ & 19 & 19 (9 months) & $<0.0001$ & 1.000 \\
\hline $2 \mathrm{~B}^{*}$ & 22 & 19 (9 months) & $<0.0001$ & 0.7 \\
\hline $2 \mathrm{C}^{*}$ & $29 \sim$ & 19 (9 months) & $<0.0001$ & 0.2 \\
\hline 3 & 51 & 75 (8 months) & 0.01 & 0.003 \\
\hline 4 & 53 & 67 (6 months) & 0.03 & 0.1 \\
\hline 5 & 51 & 75 (1 month) & 0.02 & 0.004 \\
\hline 6 & 36 & 60 (6 months) & $<0.0001$ & 0.003 \\
\hline 7 & 51 & 59 (3 months) & 0.02 & 0.4 \\
\hline 8 & 34 & 46 (6 months) & $<0.0001$ & 0.2 \\
\hline
\end{tabular}

* Center 2 ran extracts on three instruments (A-C) after watching the video. Original performance success was $7 \%$ and the instrument failed a few days later requiring preventative maintenance and part replacement. Data shown here is from the same extracts tested on the instrument after service and part replacement.

\section{Instrument Optimization Alone Was Not Sufficient to Obtain Optimal Identification}

Because spectra acquired on the NIH instrument continued to demonstrate better performance regardless of the origin of the protein extract, remote instrument service sessions were conducted with two participating centers in a pilot analysis to evaluate the effect of readjusting instrument settings such as the detector gain, ion source voltage, lens voltage, pulsed ion extraction time, and laser attenuator offset beyond adjustments made during routine preventative maintenance visits. Potential differences in extraction technique were controlled through the distribution of fresh frozen suspensions that had been extracted at the NIH. No significant difference was observed after instrument optimization in that specific circumstance (Supplementary Table 1).
Furthermore, during follow up investigation, spectra acquired directly from fresh frozen extracts using the standard MBT_AutoX method on an instrument at Bruker US headquarters illustrated reproducibility of NIH results (100 vs. 94\%, $p$-value 1.0; Supplementary Table 1), demonstrating the possibility of equivalent performance on a non-NIH instrument using the NIH database. This suggested that additional parameters, other than those that had been adjusted in this pilot analysis, may be critical for optimal mold identification. Notably, the instrument at Bruker US headquarters was used less frequently than instruments in clinical labs that run many samples per day on a continuous basis. This may have contributed to a state of better overall maintenance and thus better spectral quality and reproducibility. At several institutions, differences in performance were noted over time on a single instrument (Supplementary Figure 3). 


\section{Identification of Key Spectral Acquisition and Processing Parameters for Optimized Mold Identification}

A side-by-side comparison of spectral acquisition and processing parameters identified three key settings that repeatedly played a role in performance success in many cases (Supplementary Table 2). Modification of settings from the default Bruker MBT_AutoX method (used routinely for bacterial and yeast identification) to the settings of the NIH acquisition method or to those of an alternative method (Alternate-B method) based on this analysis, demonstrated improvement in some cases in a small-scale (six extracts) pilot analysis at five non-NIH study sites (Supplementary Figure 4). For some centers, the change in acquisition method resulted in significant improvement. For Center 5, only a change to the NIH acquisition method resulted in improved performance; equivalent performance was observed with the Alternate-B method.

To verify that use of the NIH method and the Alternate$B$ method could improve mold identification, a final challenge analysis was undertaken. Here, the full set of 80 isolates were subbed fresh and re-extracted at Center 1.71 organisms remained viable from which frozen extracts were sent to three of the original testing centers, and two additional study sites (total of nine microflex LT instruments). Detailed instructions and screenshots for programming the NIH method and AlternateB method were distributed to each center (Supplementary Figures 1, 2) and the sample plate, at each center, was run consecutively using each of the three acquisition methods (MBT_AutoX, NIH method, and Alternate-B method). A freshly spotted plate was used for each instrument. Although statistical significance between acquisition methods was achieved only on instrument 1B (Figure 1), wide performance variability remained across instruments for the MBT_AutoX acquisition method (39-85\%, p-value 0.0001). Intra-laboratory variability was also observed in Centers 1,2 , and 9 where multiple instruments were tested despite controlling for operator, isolates, technique, extraction, and methods within each respective institution. This demonstrates that multiple controllable variables contribute to the successful and reproducible performance of MALDI-TOF MS for mold identification but that instrument age and condition may also contribute to variation observed between centers and between instruments within a single center. Data suggests that the amount of improvement observed with the use of modified acquisition methods might be greater if instrument performance is not optimized (for example, contrast Centers 3 and 7 in Supplementary Figure 4; and Centers 1B and 4 in Figure 1). We propose that evaluation on a larger and diverse fungal dataset on many instruments would confirm these findings.

\section{DISCUSSION}

MALDI-TOF MS is an excellent tool for the rapid and accurate identification of molds (Alanio et al., 2011; Cassagne et al., 2011; Theel et al., 2011; Alshawa et al., 2012; De Carolis et al., 2012; Del Chierico et al., 2012; Schrodl et al., 2012; de Respinis et al., 2013; Lau et al., 2013; L'Ollivier et al., 2013; Nenoff et al., 2013;
Normand et al., 2013, 2017; Barker et al., 2014; Becker et al., 2014, 2019; Gautier et al., 2014; Ranque et al., 2014; Sitterle et al., 2014; Dolatabadi et al., 2015; Triest et al., 2015; Sleiman et al., 2016; Stein et al., 2018; Dupont et al., 2019), yet its wider application in the clinical setting has been extremely limited, in part due to limited database availability and lack of standardized processes. Here, we evaluated the versatility of the NIH Mold Database (Lau et al., 2013) across 10 centers each with varying levels of mass spectrometry experience with molds, using a set of 80 challenge isolates that encompassed common clinical molds and less common organisms (Table 1). Figure 2 provides a summary of optimization steps that laboratories may apply if mold identification using the routine setting on their instrument is suboptimal.

The wide performance variation between centers demonstrated in this study (Figure $\mathbf{1}$ and Tables 2, 3) was surprising given that high inter- and intra-laboratory reproducibility has been achieved for bacterial and yeast studies (Westblade et al., 2015; Wilson et al., 2017). We showed, however, that application of different spectral acquisition parameters $(\mathrm{NIH}$ and Alternate-B methods), along with optimized instrument tuning and maintenance, improved performance in several cases (Figure 1 and Supplementary Figure 4), suggesting that acquisition parameters are one of the key limiting variables in achieving successful performance. Comparison of acquisition methods (Supplementary Table 2) demonstrated that the minimum intensity threshold varied considerably between the MBT_AutoX, NIH, and Alternate-B methods. This parameter relates to acceptability criteria for peptide and protein peaks from molds, which are more difficult to lyse than bacteria and yeast. Interestingly, Normand et al. (2017) also identified wide performance variation between five sites that evaluated an online database for spectra acquired by the Bruker MBT system. In that study, differences were attributed to variations in sample preparation, matrix quality, and functions of the different mass spectrometers (same microflex LT model but different age and usage). Similar intra-instrument variability findings and inherent reproducibility problems were also found in a Canadian multicenter study (Stein et al., 2018). These previous publications differ to ours because they challenged different databases and studied incoming real-time samples as opposed to a standardized comparative set as used in this study. Furthermore, extraction technique and operator experience were also controlled for in our study for defined experiments. Therefore, the reproducibility of inter- and intra-instrument variability observed now across several multicenter studies that target different aspects of the process clearly suggests that MALDI-TOF MS (at least on the Bruker platform) requires optimization for mold identification, and that spotting in replicates (up to quadruplicates as proposed by Gautier et al., 2014) is not ideal. Here, we show for the first time that optimizing acquisition parameters may potentially reduce inter-operator and inter-instrument variation. In fact, the most recently released Bruker RUO software (v3.0, August 2019) has provided adjusted acquisition parameters and adapted thresholds to aid in mold identification. Given its recent availability, it is yet to be seen whether these manufacturer updates will provide improved performance for mold identification within the clinical 


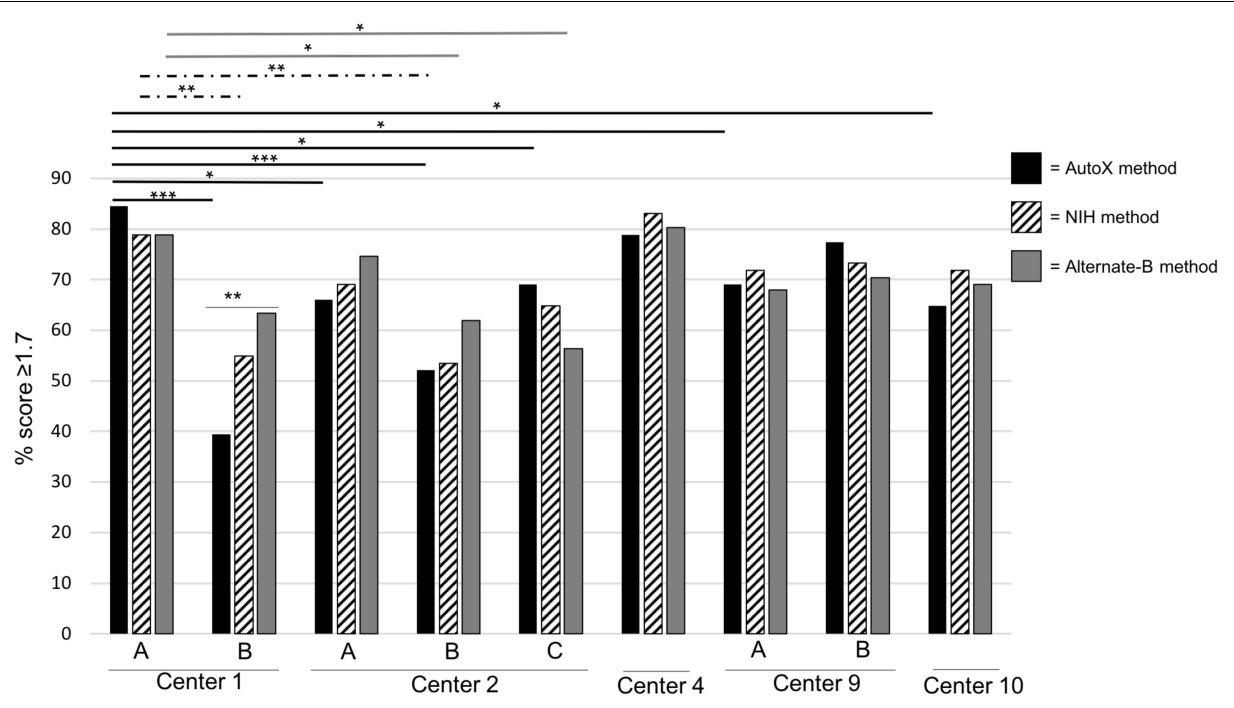

FIGURE 1 | Comparative Analysis of Three Different Spectral Acquisition Methods Using a Single Prepared Plate (2018). Fresh extracts for 71 isolates were prepared at Center 1 and distributed to each institution. For each instrument, a freshly spotted plate was made and spectra were acquired on the same day in this order of spectral acquisition methods: (1) MBT_AutoX method (black bars and lines); (2) NIH method (hashed bars and lines); and (3) Alternate-B method (gray bars and lines). Multiple instruments in a single center are denoted by letters. ${ }^{*} p<0.05,{ }^{* *} p<0.005,{ }^{* * *} p<0.0005$.

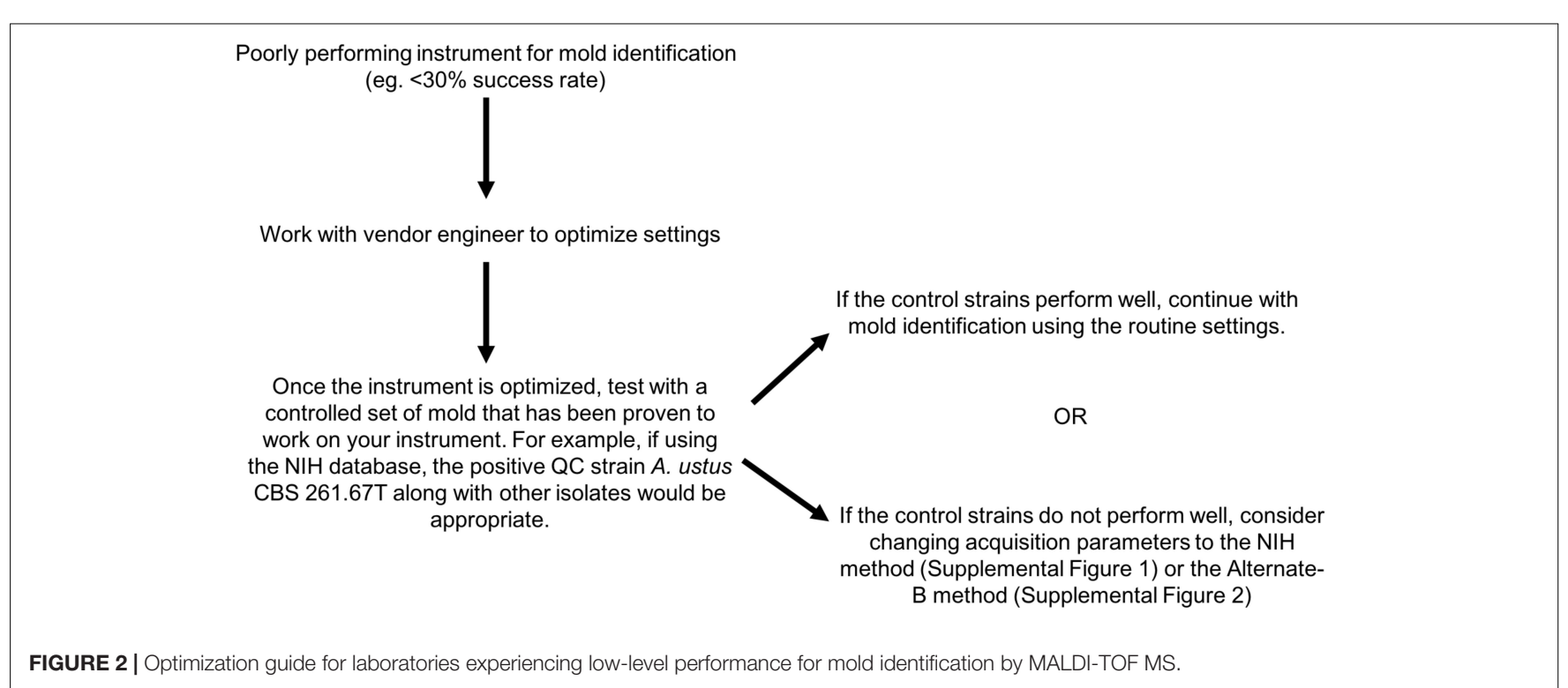

setting. In-house developed databases using the Bruker MBT system were applied in this current study and in Normand et al. (2017). In contrast, Rychert et al. (2018) reported 94\% reproducibility across three centers that evaluated the VITEK MS 3.0 (bioMerieux) platform. Importantly, the challenge sets studied in each of the multicenter evaluations varied considerably with Rychert et al. (2018) employing 50 very common clinical molds in contrast to the wider isolate diversity used in this study (Table 1) and in Normand et al. (2017). Organism challenge sets are an important consideration for reproducibility assessment and performance evaluation because MSPs for common clinical isolates are more likely to be sufficiently represented compared with rarer organisms.
While we have identified that use of modified acquisition parameters can improve performance, it is clear that other variables, potentially source cleanliness, and age of the laser and instrument components, can also affect performance (Supplementary Figure 3). These variables are instrument dependent and may require individual system optimization in addition to routine instrument calibration and maintenance. Development of a performance/calibration standard that is specific for molds and is tested during routine maintenance may be useful for instrument programming to ensure reproducibility. We propose that the A. ustus CBS 261.67T control strain be used as a marker for instrument performance, with optimal instrument performance leading to consistent log scores $>2.0$ 
for this control strain when used with the NIH Mold Database (Lau et al., 2013), even though we have demonstrated that lowering the threshold to $\geq 1.7$ for unknown patient isolates improves sensitivity without affecting accuracy. Failure of an instrument to meet these criteria would be an indication for maintenance and performance optimization. Many laboratories do not have microbiologists familiar with changing spectral acquisition parameters, and an easy to use validated method provided by the manufacturer would be ideal.

The slow adoption of MALDI-TOF MS for mold identification has been associated with the lack of supporting data and practical methods. Few investigators have evaluated the Bruker Filamentous Fungi database using the Bruker recommended protocol of liquid fungal cultures (Schulthess et al., 2014) which is not convenient for integration into routine workflow. Evaluation of the VITEK MS 3.0 (bioMerieux) has demonstrated 66.8 to $91 \%$ identification accuracy in the clinical setting, with some concerns for misidentification (McMullen et al., 2016; Rychert et al., 2018). Based on these limited data, it is not surprising that most efforts have focused on laboratorydeveloped databases which have often outperformed those of the manufacturer (Alanio et al., 2011; Cassagne et al., 2011; Theel et al., 2011; Alshawa et al., 2012; De Carolis et al., 2012; Del Chierico et al., 2012; Schrodl et al., 2012; de Respinis et al., 2013; Lau et al., 2013; L’Ollivier et al., 2013; Nenoff et al., 2013; Normand et al., 2013, 2017; Barker et al., 2014; Becker et al., 2014; Gautier et al., 2014; Ranque et al., 2014; Sitterle et al., 2014; Dolatabadi et al., 2015; Triest et al., 2015; Sleiman et al., 2016). A major limitation, however, is that all but a few studies such as this study, Normand et al. (2017), Rychert et al. (2018), and Stein et al. (2018) have been single-institution (and singleinstrument) analyses that have not addressed the significant reproducibility issues highlighted in this study. With increased implementation of MALDI-TOF MS for routine organism identification in laboratories, availability of an FDA cleared database (bioMerieux) and open access databases (Lau et al., 2013; Normand et al., 2017; Center for Disease Control and Prevention [CDCP], 2019), and with the provision of alternative spectral acquisition methods for Bruker Biotyper instruments described in this study (Supplementary Figures 1, 2) and those spectral acquisition modifications recently released by the manufacturer in updated software (v3.0), it is likely that rapid and accurate mold identification using MALDI-TOF MS can become a norm in clinical laboratories.

\section{CONCLUSION}

We have shown that the NIH Mold MALDI-TOF MS Database can be successfully transferred and implemented as a routine method for mold identification across multiple institutions if alternate spectral acquisition methods are applied along with optimization of instrument performance. A clinical laboratory initiating use of MALDI-TOF MS for mold identification would become frustrated with performance of $<30 \%$ success (e.g., Center 2A in Supplementary Figure 3), but should be reassured that $>80 \%$ success (as exemplified multiple times in this study) is possible with optimization, and they should be encouraged to work persistently with the vendor engineer until their instrument performs equivalently to those illustrated here. Continued vigilance is necessary to assure that instrument performance is maintained at the optimized level because performance can be affected by instrument use and maintenance over time. Importantly, in our experience, as instrument optimization changes over time, the result is a low score preventing mold identification rather than inaccurate identifications. Refer to Figure $\mathbf{2}$ for stepwise guide for optimizing instrument performance. More rapid and accurate identification leads to better guidance for clinicians in the selection of antifungal therapy and the collection of epidemiological data. Analysis against a larger and diverse challenge set on instruments at additional institutions is warranted. Because instrument variability is inevitable and some parameters are instrument dependent, development of an instrument performance standard specific for mold spectral acquisition is suggested to improve reproducibility across instruments.

\section{DATA AVAILABILITY}

All datasets generated for this study are included in the manuscript and/or the Supplementary Files.

\section{AUTHOR CONTRIBUTIONS}

$\mathrm{AL}$ and KF designed the study. AL, RW, HM, ES, KK, KR, AR, JG, KM, MW, ALB, UD, and CH performed the experiments and provided the data. AL, J-MB-L, BB, C-AB, SB-W, CD, KH, NL, $\mathrm{PP}, \mathrm{AS}, \mathrm{NW}, \mathrm{SZ}, \mathrm{AZ}$, and KF analyzed the data and participated in group discussions. $\mathrm{AB}, \mathrm{MK}, \mathrm{TM}$, and GS provided technical expertise on the instrument and assisted with design of the Alternate-B acquisition method. AL wrote the manuscript. All authors reviewed and edited the manuscript.

\section{FUNDING}

This work was supported by the Intramural Research Program of the National Institutes of Health. The content is solely our responsibility and does not represent the official views of the National Institutes of Health.

\section{ACKNOWLEDGMENTS}

We thank Erica Frank for assistance with video production, Adrienne Brudie for aiding in organism preparation at the New York Presbyterian hospital site, and Angella CharnotKatsikas for critical review of the manuscript.

\section{SUPPLEMENTARY MATERIAL}

The Supplementary Material for this article can be found online at: https://www.frontiersin.org/articles/10.3389/fmicb.2019. 02098/full\#supplementary-material 


\section{REFERENCES}

Alanio, A., Beretti, J. L., Dauphin, B., Mellado, E., Quesne, G., Lacroix, C., et al. (2011). Matrix-assisted laser desorption ionization time-of-flight mass spectrometry for fast and accurate identification of clinically relevant Aspergillus species. Clin. Microbiol. Infect. 17, 750-755. doi: 10.1111/j.14690691.2010.03323.x

Alshawa, K., Beretti, J.-C., Lacroix, C., Feuilhade, M., Dauphin, B., Quesne, G., et al. (2012). Successful identification of clinical dermatophyte and Neoscytalidium species by matrix-assisted laser desorption ionization-time of flight mass spectrometry. J. Clin. Microbiol. 50, 2277-2281. doi: 10.1128/JCM.06634-11

Barker, A. P., Horan, J. L., Slechta, E. S., Alexander, B. D., and Hanson, K. E. (2014). Complexities associated with the molecular and proteomic identification of Paecilomyces species in the clinical mycology laboratory. Med. Mycol. 52, 537-545. doi: 10.1093/mmy/myu001

Becker, P., Normand, A. C., Vanantwerpen, G., Vanrobaeys, M., Haesendonck, R., Vercammen, F., et al. (2019). Identification of fungal isolates by MALDI-TOF mass spectrometry in veterinary practice: validation of a web application. J. Vet. Diagn. Invest. 31, 471-474. doi: 10.1177/1040638719835577

Becker, P. T., de Bel, A., Martiny, D., Ranque, S., Piarroux, R., Cassagne, C., et al. (2014). Identification of filamentous fungi isolates by MALDI-TOF mass spectrometry: clinical evaluation of an extended reference spectra library. Med. Mycol. 52, 826-834. doi: 10.1093/mmy/myu064

Cassagne, C., Ranque, S., Normand, A. C., Fourquet, P., Thiebault, S., Planard, C., et al. (2011). Mould routine identification in the clinical laboratory by matrixassisted laser desorption ionization time-of-flight mass spectrometry. PLoS One 6:e28425. doi: 10.1371/journal.pone.0028425

Center for Disease Control and Prevention [CDCP] (2019). Prevention, C.f.D.C.a. MicrobeNet. 2019 Center for Disease Control and Prevention. Available at: https: //www.cdc.gov/microbenet/index.html (accessed September 5, 2019).

Clinical and Laboratory Standards Institute [CLSI] (2008). Clinical and Laboratory Standards Institute, Interpretive Criteria for Identification of Bacteria and Fungi by DNA Target Sequencing; Approved Guidelines (CLSI Document MM18-A). Wayne, PA: Clinical and Laboratory Standards Institute.

De Carolis, E., Posteraro, B., Lass-Florl, C., Vella, A., Florio, A. R., Torelli, R., et al. (2012). Species identification of Aspergillus, Fusarium and Mucorales with direct surface analysis by matrix-assisted laser desorption ionization time-offlight mass spectrometry. Clin. Microbiol. Infect 18, 475-484. doi: 10.1111/j. 1469-0691.2011.03599.x

de Respinis, S., Tonolla, M., Pranghofer, S., Petrini, L., Petrini, O., and Bosshard, P. P. (2013). Identification of dermatophytes by matrix-assisted laser desorption/ionization time-of-flight mass spectrometry. Med. Mycol. 51, 514-521. doi: 10.3109/13693786.2012.746476

Del Chierico, F., Masotti, A., Onori, M., Fiscarelli, E., Mancinelli, L., Ricciotti, G., et al. (2012). MALDI-TOF MS proteomic phenotyping of filamentous and other fungi from clinical origin. J. Proteom. 75, 3314-3330. doi: 10.1016/j.jprot.2012. 03.048

Dolatabadi, S., Kolecka, A., Versteeg, M., de Hoog, S. G., and Boekhout, T. (2015). Differentiation of clinically relevant Mucorales Rhizopus microsporus and R. arrhizus by matrix-assisted laser desorption ionization time-of-flight mass spectrometry (MALDI-TOF MS). J. Med. Microbiol. 64, 694-701. doi: 10.1099/jmm.0.000091

Dupont, D., Normand, A. C., Persat, F., Hendrickx, M., Piarroux, R., and Wallon, M. (2019). Comparison of matrix-assisted laser desorption ionization time of flight mass spectrometry (MALDI-TOF MS) systems for the identification of moulds in the routine microbiology laboratory. Clin. Microbiol. Infect. 25, 892-897. doi: 10.1016/j.cmi.2018.10.013

Gautier, M., Ranque, S., Normand, A. C., Becker, P., Packeu, A., Cassagne, C., et al. (2014). Matrix-assisted laser desorption ionization time-of-flight mass spectrometry: revolutionizing clinical laboratory diagnosis of mould infections. Clin. Microbiol. Infect. 20, 1366-1371. doi: 10.1111/1469-0691.12750

Huang, A. M., Newton, D., Kunapuli, A., Gandhi, T. N., Washer, L. L., Isip, J., et al. (2013). Impact of rapid organism identification via matrix-assisted laser desorption/ionization time-of-flight combined with antimicrobial stewardship team intervention in adult patients with bacteremia and candidemia. Clin. Infect. Dis. 57, 1237-1245. doi: 10.1093/cid/cit498

Lacroix, C., Gicquel, A., Sendid, B., Meyer, J., Accoceberry, I., Francois, N., et al. (2014). Evaluation of two matrix-assisted laser desorption ionization-time of flight mass spectrometry (MALDI-TOF MS) systems for the identification of Candida species. Clin. Microbiol. Infect. 20, 153-158. doi: 10.1111/1469-0691. 12210

Lau, A. F., Drake, S. K., Calhoun, L. B., Henderson, C. M., and Zelazny, A. M. (2013). Development of a clinically comprehensive database and a simple procedure for identification of molds from solid media by matrix-assisted laser desorption ionization-time of flight mass spectrometry. J. Clin. Microbiol. 51, 828-834. doi: 10.1128/JCM.02852-12

L'Ollivier, C., Cassagne, C., Normand, A. C., Bouchara, J. P., Contet-Audonneau, N., Hendrickx, M., et al. (2013). A MALDI-TOF MS procedure for clinical dermatophyte species identification in the routine laboratory. Med. Mycol. 51, 713-720. doi: 10.3109/13693786.2013.781691

McMullen, A. R., Wallace, M. A., Pincus, D. H., Wilkey, K., and Burnham, C. A. (2016). Evaluation of the vitek MS matrix-assisted laser desorption ionizationtime of flight mass spectrometry system for identification of clinically relevant Filamentous fungi. J. Clin. Microbiol. 54, 2068-2073. doi: 10.1128/JCM.008 25-16

Nenoff, P., Erhard, M., Simon, J. C., Muylowa, G. K., Herrmann, J., Rataj, W., et al. (2013). MALDI-TOF mass spectrometry - a rapid method for the identification of dermatophyte species. Med. Mycol. 51, 17-24. doi: 10.3109/13693786.2012. 685186

Normand, A. C., Becker, P., Gabriel, F., Cassagne, C., Accoceberry, I., GariToussaint, M., et al. (2017). Validation of a new web application for identification of fungi by use of matrix-assisted laser desorption ionization-time of flight mass spectrometry. J. Clin. Microbiol. 55, 2661-2670. doi: 10.1128/JCM. 00263-17

Normand, A. C., Cassagne, C., Ranque, S., L'Ollivier, C., Fourquet, P., Roesems, S., et al. (2013). Assessment of various parameters to improve MALDITOF MS reference spectra libraries constructed for the routine identification of filamentous fungi. BMC Microbiol. 13:76. doi: 10.1186/1471-218013-76

Ranque, S., Normand, A. C., Cassagne, C., Murat, J. B., Bourgeois, N., Dalle, F., et al. (2014). MALDI-TOF mass spectrometry identification of filamentous fungi in the clinical laboratory. Mycoses 57, 135-140. doi: 10.1111/myc. 12115

Rychert, J., Slechta, E. S., Barker, A. P., Miranda, E., Babady, N. E., Tang, Y. W., et al. (2018). Multicenter Evaluation of the vitek MS v3.0 system for the identification of Filamentous fungi. J. Clin. Microbiol. 56:e01353-17. doi: 10.1128/JCM.013 53-17

Schrodl, W., Heydel, T., Schwartze, V. U., Hoffmann, K., Grosse-Herrenthey, A., Walther, G., et al. (2012). Direct analysis and identification of pathogenic Lichtheimia species by matrix-assisted laser desorption ionization-time of flight analyzer-mediated mass spectrometry. J. Clin. Microbiol. 50, 419-427. doi: 10. 1128/JCM.01070-11

Schulthess, B., Ledermann, R., Mouttet, F., Zbinden, A., Bloemberg, G. V., Bottger, E. C., et al. (2014). Use of the bruker MALDI biotyper for identification of molds in the clinical mycology laboratory. J. Clin. Microbiol. 52, 2797-2803. doi: 10.1128/JCM.00049-14

Sitterle, E., Giraud, S., Leto, J., Bouchara, J. P., Rougeron, A., Morio, F., et al. (2014). Matrix-assisted laser desorption ionization-time of flight mass spectrometry for fast and accurate identification of Pseudallescheria/Scedosporium species. Clin. Microbiol. Infect. 20, 929-935. doi: 10.1111/1469-0691.12574

Sleiman, S., Halliday, C. L., Chapman, B., Brown, M., Nitschke, J., Lau, A. F., et al. (2016). Performance of matrix-assisted laser desorption ionization-time of flight mass spectrometry for identification of Aspergillus, Scedosporium, and Fusarium spp. in the Australian clinical setting. J. Clin. Microbiol. 54, 2182-2186. doi: 10.1128/JCM.00906-16

Stein, M., Tran, V., Nichol, K. A., Lagace-Wiens, P., Pieroni, P., Adam, H. J., et al. (2018). Evaluation of three MALDI-TOF mass spectrometry libraries for the identification of filamentous fungi in three clinical microbiology laboratories in Manitoba. Can. Mycoses 61, 743-753. doi: 10.1111/myc. 12800

Tan, K. E., Ellis, B. C., Lee, R., Stamper, P. D., Zhang, S. X., and Carroll, K. C. (2012). Prospective evaluation of a matrix-assisted laser desorption ionization-time of flight mass spectrometry system in a hospital clinical microbiology laboratory for identification of bacteria and yeasts: a bench-by-bench study for assessing the impact on time to identification and cost-effectiveness. J. Clin. Microbiol. 50, 3301-3308. doi: 10.1128/JCM.01405-12 
Theel, E. S., Hall, L., Mandrekar, J., and Wengenack, N. L. (2011). Dermatophyte identification using matrix-assisted laser desorption ionization-time of flight mass spectrometry. J. Clin. Microbiol. 49, 4067-4071. doi: 10.1128/JCM.012 80-11

Triest, D., Stubbe, D., De Cremer, K., Pierard, D., Normand, A. C., Piarroux, R., et al. (2015). Use of matrix-assisted laser desorption ionization-time of flight mass spectrometry for identification of molds of the Fusarium genus. J. Clin. Microbiol. 53, 465-476. doi: 10.1128/JCM.02213-14

United States Food and Drug Administration (2017). The 510(k) Document K162950: Substantial Equivalence Determination Decision Summary, 2017. Maryland, MD: United States Food, and Drug Administration.

Westblade, L. F., Garner, O. B., MacDonald, K., Bradford, C., Pincus, D. H., Mochon, A. B., et al. (2015). Assessment of reproducibility of matrix-assisted laser desorption ionization-time of flight mass spectrometry for bacterial and yeast identification. J. Clin. Microbiol. 53, 2349-2352. doi: 10.1128/JCM.001 $87-15$

White, T. J., Bruns, T. D., Lee, S. B., and Taylor, J. W. (1990). "Amplification and direct sequencing of fungal ribosomal RNA genes for phylogenetics," in PCR Protocols: A Guide to Methods and Applications, eds M. A. Innis, D. H. Gelfand, J. J. Sninsky, and T. J. White (New York, NY: Academic Press, Inc), 315-322. doi: 10.1016/b978-0-12-372180-8.50042-1

Wilson, D. A., Young, S., Timm, K., Novak-Weekley, S., Marlowe, E. M., Madisen, N., et al. (2017). Multicenter evaluation of the bruker MALDI biotyper CA system for the identification of clinically important bacteria and yeasts. Am. J. Clin. Pathol. 147, 623-631. doi: 10.1093/ajcp/aqw225
Conflict of Interest Statement: AL, AZ, and KF were previously part of a collaborative agreement with Bruker Daltonics, Inc. for the development of microbial databases. KR, BB, and NL have received research funding from both Bruker Daltonics, Inc. and bioMerieux to conduct clinical trials. PP has received research funding from Bruker Daltonics, Inc. to conduct clinical trials. $\mathrm{C}-\mathrm{AB}$ has received research funding from bioMerieux and speaker fees from Bruker Daltonics, Inc. AB, MK, TM, and GS are employees of Bruker Daltonics, Inc., the manufacturer of the MALDI-TOF MS system used in this study; Bruker Daltonics, Inc. had no influence on the design of the study, data collection, or the interpretation of the study results.

The remaining authors declare that the research was conducted in the absence of any commercial or financial relationships that could be construed as a potential conflict of interest.

Copyright (c) 2019 Lau, Walchak, Miller, Slechta, Kamboj, Riebe, Robertson, Gilbreath, Mitchell, Wallace, Bryson, Balada-Llasat, Bulman, Buchan, Burnham, Butler-Wu, Desai, Doern, Hanson, Henderson, Kostrzewa, Ledeboer, Maier, Pancholi, Schuetz, Shi, Wengenack, Zhang, Zelazny and Frank. This is an openaccess article distributed under the terms of the Creative Commons Attribution License (CC BY). The use, distribution or reproduction in other forums is permitted, provided the original author(s) and the copyright owner(s) are credited and that the original publication in this journal is cited, in accordance with accepted academic practice. No use, distribution or reproduction is permitted which does not comply with these terms. 